\title{
artigo
}

Oliveira, B.S.; Aguiar, R.S.

Repercussões na saúde da mulher adolescente devido a gravidez precoce: uma revisão narrativa

\section{Repercussões na saúde da mulher adolescente devido a gravidez precoce: uma revisão narrativa}

\author{
Repercussions on the health of adolescent women due to early pregnancy: a narrative review \\ Repercusiones sobre la salud de mujeres adolescentes debido a embarazo temprano: una revisión narrativa
}

\begin{abstract}
RESUMO
Objetivo: Discutir, a partir de uma reflexão teórica, as repercussões na saúde da mulher adolescente devido a gravidez precoce. Métodos: Trata-se de um estudo qualitativo, descritivo do tipo teórico-reflexivo desenvolvido a partir da abordagem de revisão narrativa com artigos científicos nacionais e internacionais disponíveis nas bases de dados LILACS, PUBMED e SciELO. Resultados: Entre as repercussões decorrentes da gravidez na adolescência que a mulher pode estar exposta, identificou-se: complicações obstétricas, aborto inseguro, síndromes hipertensivas e quadros depressivos. Além disso, questões sociodemográficas, início precoce da vida sexual e ausência de planejamento reprodutivo estão entre os fatores associados à ocorrência da gravidez na adolescência. Conclusão: Os achados da literatura apontam uma leve queda da gravidez na adolescência no Brasil, mas os números ainda são altos. Apesar do entendimento dos profissionais de saúde sobre a importância de programas de educação sexual para as famílias, ainda falta um acolhimento eficiente para o público jovem.
\end{abstract}

DESCRITORES: Gravidez na adolescência; Complicações na gravidez; Parto; Peso ao nascer; Maturidade sexual.

\section{ABSTRACT}

Objective: To discuss, from a theoretical reflection, the repercussions on the health of adolescent women due to early pregnancy. Methods: This is a qualitative, descriptive study of the theoretical-reflective type developed from the narrative review approach with national and international scientific articles available in the LILACS, PUBMED and SciELO databases. Results: Among the repercussions resulting from teenage pregnancy that women may be exposed to, it was identified: obstetric complications, unsafe abortion, hypertensive syndromes and depressive conditions. In addition, sociodemographic issues, early onset of sexual life and lack of reproductive planning are among the factors associated with the occurrence of teenage pregnancy. Conclusion: The findings in the literature point to a slight fall in teenage pregnancy in Brazil, but the numbers are still high. Despite the understanding of health professionals about the importance of sex education programs for families, there is still an lack of efficient reception for young people.

DESCRIPTORS: Pregnancy in adolescence; Pregnancy complications; Parturition; Birth weight; Sexual maturation.

\section{RESUMEN}

Objetivo: Discutir, desde una reflexión teórica, las repercusiones en la salud de la mujer adolescente por el embarazo precoz. Métodos: Se trata de un estudio cualitativo, descriptivo de tipo teórico-reflexivo desarrollado a partir del enfoque de revisión narrativa con artículos científicos nacionales e internacionales disponibles en las bases de datos LILACS, PUBMED y SciELO. Resultados: Entre las repercusiones derivadas del embarazo adolescente a las que pueden estar expuestas las mujeres, se identificaron: complicaciones obstétricas, aborto inseguro, síndromes hipertensivos y condiciones depresivas. Además, las cuestiones sociodemográficas, el inicio temprano de la vida sexual y la falta de planificación reproductiva se encuentran entre los factores asociados con la ocurrencia del embarazo adolescente. Conclusión: Los hallazgos en la literatura apuntan a una leve caída en el embarazo adolescente en Brasil, pero las cifras siguen siendo altas. A pesar de la comprensión de los profesionales de la salud sobre la importancia de los programas de educación sexual para las familias, aún existe una falta de recepción eficiente para los jóvenes.

DESCRIPTORES: Embarazo em adolescencia; Complicaciones del embarazo; Parto; Peso al nacer; Maturación sexual.

RECEBIDO EM: 03/04/2021 APROVADO EM: 23/04/2021

\section{Bruna de Souza Oliveira}

Acadêmica de Enfermagem. Universidade Paulista (UNIP), Campus Brasília-DF. Brasília-DF, Brasil.

ORCID: 0000-0003-1171-4060 


\section{Ricardo Saraiva Aguiar}

Professor Assistente. Curso de Graduação em Enfermagem. Universidade Paulista (UNIP), Campus Brasília-DF.

ORCID: 0000-0003-0335-2194

\section{INTRODUÇÃO}

A adolescência compreende uma etapa de importantes mudanças físicas psíquicas, correspondendo em um período de transformações e de modificações entre a vivência infantil na qual $\mathrm{o}(\mathrm{a})$ adolescente está acostumado(a) e uma nova fase chamada adolescência, que não tendo o acompanhamento necessário pode resultar em consequências que repercutirão por toda a vida. ${ }^{1-2}$

No Brasil, esta etapa compreende o período de idade entre 12 e 18 anos de acordo com o Estatuto da Criança e do Adolescente (ECA) e é caracterizada como uma transição da infância para a fase adulta que terá como consequência a mudança do indivíduo ao desenvolver alterações físicas, mentais, emocionais, sexuais, sociais e de formação da própria identidade. Toda essa fase de transformação é caracterizada pelo processo de puberdade e este processo só acaba quando o indivíduo finaliza a fase de crescimento, criando sua identidade social. ${ }^{3}$

Nessa perspectiva, a educação sexual durante a adolescência é imprescindível devido a necessidade de $\mathrm{o}(\mathrm{a})$ adolescente adquirir a segurança necessária, perceber que sua vida sexual está se iniciando e que dispõe de amparo - seja da família, dos professores ou dos profissionais de saúde - para receberem informações oportunas sobre o assunto ${ }^{4}$, pois a taxa de gravidez na adolescência no Brasil é superior à mundial e entre 2010 e 2015, segundo dados da Organização Mundial da Saúde (OMS), a taxa mundial de gravidez na adolescência foi de 46 por 1.000 meninas. $^{5}$

Devido a diversos fatores que podem influenciar a gravidez na adolescência, o assunto tornou-se uma preocupação de saúde pública devido as complicações que podem ocasionar para a saúde das jovens mães e de seus filhos. ${ }^{6}$ Destacam-se as complicações obstétricas, mas apresenta-se também como problemáticas a questão
No Brasil, esta etapa compreende o período de idade entre $12 \mathrm{e}$

\section{8 anos de acordo}

com o Estatuto

da Criança e do

Adolescente (ECA)

e é caracterizada

como uma transição

da infância para a

fase adulta que terá

como consequência

a mudança do

indivíduo ao

desenvolver

alterações físicas,

mentais, emocionais,

sexuais, sociais e de

formação da própria

identidade. do aborto ilegal e inseguro, anemia, desnutrição, sobrepeso, hipertensão, dentre outras. ${ }^{6-7}$

Precárias condições socioeconômicas, associadas à dificuldade de acesso aos serviços e à informação adequada contribuem para o problema. No sentido de desenvolver medidas de enfrentamento desse fenômeno, os profissionais de saúde têm significativa importância, visto que ocupam lugares estratégicos em todos os níveis de atenção à saúde. Além disso, podem estar presentes não somente em serviços específicos de saúde, mas também em outros ambientes nos quais convivem adolescentes, especialmente, a escola. ${ }^{7}$

Contudo, a ausência ou ineficiência de abordagens estratégicas e ações prioritárias sobre essa temática em populações comumente negligenciadas são questões que tornam a adolescente ainda mais vulnerável no que diz respeito ao direito à vida e à saúde. A gravidez afeta eminentemente as trajetórias dessas vidas ao impulsionar as meninas à maternidade antes de estarem preparadas física, emocional ou financeiramente, por vezes perpetuando os ciclos intergeracionais de pobreza. ${ }^{8}$

Assim, é necessário a participação de toda uma equipe multiprofissional no atendimento à adolescente de modo a planejar estratégias e intervenções de educação sexual para evitar a gestação precoce neste público. ${ }^{6}$

Portanto, o presente estudo tem o objetivo de discutir, a partir de uma reflexão teórica, as repercussões na saúde da mulher adolescente devido a gravidez precoce. Sob essa perspectiva, destaca-se a questão que norteou a presente proposta investigativa: quais as repercussões na saúde da adolescente devido a gravidez precoce?

\section{MÉTOdOS}

Trata-se de um estudo qualitativo, descritivo do tipo teórico-reflexivo desenvolvido a partir da abordagem de revisão 


\section{artigo}

Oliveira, B.S.; Aguiar, R.S.

Repercussões na saúde da mulher adolescente devido a gravidez precoce: uma revisão narrativa

narrativa com artigos científicos nacionais e internacionais que abordam sobre as repercussões na saúde da mulher adolescente em decorrência da gravidez precoce.

Os artigos de revisão narrativa são publicações amplas apropriadas para descrever e discutir o desenvolvimento ou o estado da arte de um determinado assunto, sob o ponto de vista teórico ou conceitual. São textos que constituem a análise da literatura científica na interpretação e análise crítica do autor. ${ }^{9}$

Realizou-se, para a busca das produçôes, a seguinte pergunta de pesquisa: "Quais as repercussões na saúde da adolescente devido a gravidez precoce?". Selecionaram-se, assim, os artigos que abordavam a gravidez na adolescência e repercussões na saúde da mulher adolescente.
Os artigos foram pesquisados no período de agosto a outubro de 2020 por meio das bases de dados Literatura Latino-Americana e do Caribe em Ciências da Saúde (LILACS), PUBMED e Scientific Eletronic Library Online (SciELO), refinando a busca para o período de 2006 a 2019 e utilizando os seguintes critérios de inclusão: artigos disponíveis de forma online; no idioma português, inglês ou espanhol; e pesquisas originais ou de revisão de literatura.

Dividiu-se o estudo em etapas: pesquisa dos artigos nas bases de dados; leitura dos títulos e dos resumos para verificar a convergência do material à temática de estudo e aos critérios de inclusão; leitura do artigo na íntegra; busca e leitura dos estudos originais encontrados por meio das referências finais dos artigos oriundos da busca nas bases de dados. Realizaram-se, após todas as leituras, a compilação dos materiais, seguida da análise sobre as repercussões na saúde da mulher adolescente em decorrência da gravidez precoce para a reflexão e, finalmente, a elaboração das sínteses reflexivas do estudo.

Com os artigos selecionados, utilizou-se a síntese narrativa para análise e discussão dos dados.

\section{RESULTADOS}

Foram utilizados 11 artigos nesta revisão narrativa (Quadro 1), sendo interpretados e sintetizados os resultados através de uma comparação dos dados evidenciados na análise dos artigos.

Quadro 1 - Distribuição dos artigos de acordo com o periódico, ano de publicação, autor(es), título, delineamento e resultados. Brasilia, Distrito Federal, Brasil, 2020.

\begin{tabular}{|c|c|c|c|c|c|}
\hline & $\begin{array}{l}\text { PERIÓDICO E ANO } \\
\text { DE PUBLICAÇÃO }\end{array}$ & AUTOR(ES) & TÍTULO & DELINEAMENTO & RESULTADOS \\
\hline 㝘 & $\begin{array}{c}\text { Revista da } \\
\text { Associação Médica } \\
\text { Brasileira, } 2019\end{array}$ & $\begin{array}{c}\text { Monteiro DLM, } \\
\text { Martins JAFS, } \\
\text { Rodrigues NCP, } \\
\text { Miranda FRD, } \\
\text { Lacerda IMS, } \\
\text { Souza FM et al. }\end{array}$ & $\begin{array}{l}\text { Adolescent pregnancy } \\
\text { trends in the last decade }\end{array}$ & $\begin{array}{l}\text { Estudo } \\
\text { Epidemiológico }\end{array}$ & $\begin{array}{l}\text { A gravidez na adolescência no Brasil } \\
\text { está em lento declínio, principalmente } \\
\text { entre as mães de } 10 \text { a } 14 \text { anos, e está } \\
\text { associada ao IDH, exceto no Nordeste. }\end{array}$ \\
\hline 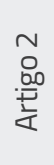 & $\begin{array}{l}\text { Cadernos Saúde } \\
\text { Coletiva, } 2019\end{array}$ & $\begin{array}{l}\text { Pinheiro YT, } \\
\text { Pereira NH, } \\
\text { Freitas GDM }\end{array}$ & $\begin{array}{l}\text { Fatores associados à } \\
\text { gravidez em adolescentes } \\
\text { de um município do } \\
\text { nordeste do Brasil }\end{array}$ & Transversal & $\begin{array}{l}\text { A gravidez na adolescência está } \\
\text { associada a condicionalidades } \\
\text { sociodemográficas, de comportamento } \\
\text { sexual e de planejamento familiar. }\end{array}$ \\
\hline $\begin{array}{l}m \\
0 \\
\frac{00}{1} \\
\frac{1}{4}\end{array}$ & $\begin{array}{l}\text { Revista Brasileira } \\
\text { de Ginecologia e } \\
\text { Obstetrícia, } 2009\end{array}$ & $\begin{array}{l}\text { Santos GHN, } \\
\text { Martins MG, } \\
\text { Sousa MS, } \\
\text { Batalha SJC }\end{array}$ & $\begin{array}{l}\text { Impacto da idade materna } \\
\text { sobre os resultados } \\
\text { perinatais e via de parto }\end{array}$ & Quantitativo & $\begin{array}{l}\text { A gravidez na adolescência esteve } \\
\text { associada a início tardio e menor } \\
\text { número de consultas o pré-natal, uso } \\
\text { de abortivo no início da gestação, baixa } \\
\text { escolaridade, ausência de companheiro, } \\
\text { baixo peso ao nascer, prematuridade } \\
\text { e menor incidência de desproporção } \\
\text { céfalo-pélvica e pré-eclâmpsia. }\end{array}$ \\
\hline 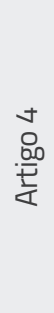 & $\begin{array}{c}\text { Revista Brasileira } \\
\text { de Saúde Materno } \\
\text { Infantil, } 2017\end{array}$ & $\begin{array}{l}\text { Bacelar EB, } \\
\text { Costa COM, } \\
\text { Gama SGN, } \\
\text { Amaral MTR, } \\
\text { Almeida AHV }\end{array}$ & $\begin{array}{l}\text { Fatores associados à } \\
\text { Síndrome Hipertensiva } \\
\text { Específica da Gestação } \\
\text { em puérperas } \\
\text { adolescentes e adultas } \\
\text { jovens da região Nordeste } \\
\text { do Brasil: análise múltipla } \\
\text { em modelos hierárquicos }\end{array}$ & Transversal & $\begin{array}{l}\text { Identificou-se associações significativas } \\
\text { entre SHEG e puérperas adolescentes } \\
\text { e adultas jovens sem companheiro, } \\
\text { com baixa escolaridade e antecedentes } \\
\text { clínicos de risco. }\end{array}$ \\
\hline
\end{tabular}




\begin{tabular}{|c|c|c|c|c|c|}
\hline 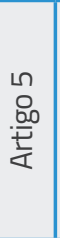 & Revista Rene, 2010 & $\begin{array}{l}\text { Silva MP, } \\
\text { Santos ZMSA, } \\
\text { Nascimento RO, } \\
\text { Fonteles JL }\end{array}$ & $\begin{array}{l}\text { Avaliação das condutas } \\
\text { de prevenção da } \\
\text { Síndrome Hipertensiva } \\
\text { Específica da Gravidez } \\
\text { entre adolescentes }\end{array}$ & Quantitativo & $\begin{array}{l}\text { As adolescentes informaram precárias } \\
\text { condições socioeconômicas, baixa } \\
\text { escolaridade, riscos para a ocorrência } \\
\text { da SHEG, fragmentação do saber e } \\
\text { autocuidado inadequado em relação à } \\
\text { prevenção deste agravo. }\end{array}$ \\
\hline 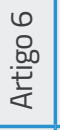 & $\begin{array}{c}\text { Revista Chilena } \\
\text { de Obstetrícia y } \\
\text { Ginecologia, } 2018\end{array}$ & $\begin{array}{l}\text { Parra EJL, } \\
\text { Herrada CHL, } \\
\text { Parra DML }\end{array}$ & $\begin{array}{l}\text { El recién-nascido de } \\
\text { madre adolescente }\end{array}$ & Retrospectivo & $\begin{array}{l}\text { A maioria dos recém-nascidos } \\
\text { nasceram a termo e por cesariana. Não } \\
\text { houve mortes. }\end{array}$ \\
\hline 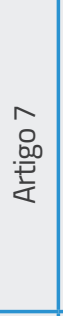 & $\begin{array}{c}\text { Revista de } \\
\text { Psiquiatria Clínica, } \\
2010\end{array}$ & $\begin{array}{c}\text { Pereira PK, } \\
\text { Lovisi GM, Lima } \\
\text { LA, Legay LF }\end{array}$ & $\begin{array}{l}\text { Complicações obstétricas, } \\
\text { eventos estressantes, } \\
\text { violência e depressão } \\
\text { durante a gravidez em } \\
\text { adolescentes atendidas } \\
\text { em unidade básica de } \\
\text { saúde }\end{array}$ & Quantitativo & $\begin{array}{l}\text { A prevalência de depressão gestacional } \\
\text { foi de } 14,2 \% \text { sendo os principais } \\
\text { fatores associados: história anterior } \\
\text { de depressão, sangramento anômalo } \\
\text { e hospitalização na atual gravidez, } \\
\text { história de acidente, incêndio ou } \\
\text { catástrofe e maus-tratos durante a } \\
\text { vida. }\end{array}$ \\
\hline 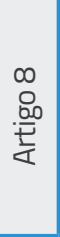 & $\begin{array}{l}\text { Psicologia: teoria e } \\
\text { pesquisa, } 2019\end{array}$ & $\begin{array}{l}\text { Frizzo GB, } \\
\text { Martins LWF, } \\
\text { Silva EXL, } \\
\text { Piccinini CA, } \\
\text { Diehl AMP }\end{array}$ & $\begin{array}{l}\text { Maternidade adolescente: } \\
\text { a matriz de apoio e o } \\
\text { contexto de depressão } \\
\text { pós-parto }\end{array}$ & Qualitativo & $\begin{array}{l}\text { O fato das participantes estarem } \\
\text { vivenciando a adolescência } \\
\text { concomitantemente à maternidade } \\
\text { pode potencializar as ambivalências } \\
\text { características dessas etapas } \\
\text { desenvolvimentais. }\end{array}$ \\
\hline 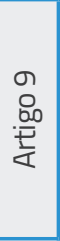 & $\begin{array}{l}\text { Revista Brasileira } \\
\text { de Saúde Materno } \\
\text { Infantil, } 2014\end{array}$ & $\begin{array}{l}\text { Almeida AHV, } \\
\text { Costa COM, } \\
\text { Gama SGN, } \\
\text { Amaral MTR, } \\
\text { Vieira GP }\end{array}$ & $\begin{array}{c}\text { Baixo peso ao nascer em } \\
\text { adolescentes e adultas } \\
\text { jovens na região nordeste } \\
\text { do Brasil }\end{array}$ & Transversal & $\begin{array}{l}\text { Alta frequência de baixo peso ao nascer } \\
\text { entre mães adolescentes e adultas } \\
\text { jovens, tendo como principais fatores } \\
\text { associados o número insuficiente de } \\
\text { consultas pré-natal, primigestação e } \\
\text { prematuridade. }\end{array}$ \\
\hline $\begin{array}{l}\frac{0}{0} \\
0 \\
.00 \\
\frac{.00}{2}\end{array}$ & $\begin{array}{l}\text { Revista Brasileira } \\
\text { de Saúde Materno } \\
\text { infantil, } 2006\end{array}$ & $\begin{array}{l}\text { Carniel EF, } \\
\text { Zanolli ML, } \\
\text { Almeida CAA, } \\
\text { Morcilio AM }\end{array}$ & $\begin{array}{l}\text { Características das mães } \\
\text { adolescentes e de seus } \\
\text { recém-nascidos e fatores } \\
\text { de risco para a gravidez } \\
\text { na adolescência em } \\
\text { Campinas, SP, Brasil }\end{array}$ & Transversal & $\begin{array}{l}\text { O perfil das mães adolescentes e de } \\
\text { seus partos e os fatores de risco para } \\
\text { gravidez na adolescência relacionaram- } \\
\text { se principalmente com condições } \\
\text { socioeconômicas desfavoráveis, } \\
\text { sugerindo que as intervenções } \\
\text { requerem ações intersetoriais. }\end{array}$ \\
\hline $\begin{array}{l}5 \\
\frac{5}{0} \\
\text { 尊 } \\
\frac{1}{4}\end{array}$ & $\begin{array}{l}\text { Revista Brasileira } \\
\text { de Ginecologia e } \\
\text { Obstetrícia, } 2009\end{array}$ & $\begin{array}{c}\text { Silva JLCP, Surita } \\
\text { FGC }\end{array}$ & $\begin{array}{l}\text { Idade materna: resultados } \\
\text { perinatais e via de parto }\end{array}$ & Reflexão & $\begin{array}{c}\text { Para as mulheres com mais de } 35 \text { anos, } \\
\text { é estabelecido que estas constituem } \\
\text { um grupo de risco bem determinado, o } \\
\text { qual demanda cuidados especializados, } \\
\text { igualmente multiprofissionais } \\
\text { na perspectiva das complicações } \\
\text { peculiares associadas à idade, e há uma } \\
\text { tendência de incremento em todos os } \\
\text { extratos sociais. }\end{array}$ \\
\hline & veira BS, Aguiar RS, 2020. & & & & \\
\hline
\end{tabular}

\section{DISCUSSÃO}

A gravidez na adolescência é de ocorrência mundial, mas prevalece com maior frequência nos países em desenvolvimento. Ao avaliar a taxa de fecundidade no Brasil, a partir dos anos 2000 inicia-se um declínio no índice de gravidez neste público, mas os números ainda continuam elevados. De acordo com dados do SINASC, entre 2006 e 2015 houve diminuição nos índices de gravidez em adolescentes na faixa de idade de 10 a 14 anos, mas na região Nordeste do país, ainda não há apontamentos de de- clínio, representando apenas $4 \%$ de queda neste período avaliado. ${ }^{10}$

Diante disso, a ocorrência da gravidez na adolescência está, muitas vezes, vinculada a questões sociodemográficas, início precoce da vida sexual e de ausência de planejamento reprodutivo. Comumente, as gestações 
não planejadas em adolescentes causam riscos de complicações obstétricas a mãe e ao filho pelo desconhecimento da gestante adolescente sobre a gravidez e o seu comportamento em relação ao consumo de medicamentos, álcool, tabaco e outras drogas no início da gestação. ${ }^{11}$

Ademais, as complicações advindas da gravidez na adolescência podem estar associadas também ao início tardio do pré-natal, bem como pela ausência nas consultas de rotina e pela falta de apoio do companheiro. ${ }^{12}$

Partos prematuros e anemia estão associados à gestação na adolescência. Além disso, ao engravidar precocemente a adolescente pode apresentar maior risco de apresentar Síndrome Hipertensiva Específica da Gravidez (SHEG), que é uma complicação frequente e um fator de risco de vida para mãe e para a criança, devido a complicações associadas à pré-eclâmpsia e eclâmpsia. ${ }^{13}$ A SHEG pode ser mais comum em adolescentes grávidas menores de 17 anos por questôes específicas da idade, como a imaturidade de órgãos e sistemas. ${ }^{14}$

Em pesquisa realizada no Nordeste do Brasil, identificou-se uma prevalência de SHEG em 4,6\% das adolescentes. A presença de hipertensão arterial esteve presente no grupo de adolescentes grávidas e foi um fator de risco de vida materna e neonatal. Dos fatores que podem interferir para ocorrência de uma SHEG, foi possível observar que situações sociais e biológicas também influenciaram significativamente, como ausência de um companheiro, a baixa escolaridade e a presença de antecedentes clínicos. ${ }^{13}$

Ao analisar 644 recém-nascidos de mães adolescentes, observou-se que 97,2\% dos partos tiveram apresentação cefálica, mas cerca de $61 \%$ dos casos analisados necessitaram realizar o parto cesáreo. Além disso, a partir do diagnóstico pediátrico do prontuário, cerca de $10 \%$ dos recém-nascidos eram pequenos para idade gestacional. ${ }^{15}$

Em um estudo epidemiológico seccional com entrevistas realizadas em consultas de pré-natal de adolescentes entre $15 \mathrm{e}$ 19 anos foi possível observar que $16 \%$ das adolescentes referiram história anterior de depressão, sendo associado a depressão na gravidez. Ademais, 33\% referiram confli- tos pessoais com pessoas próximas; $31,7 \%$ afirmaram estar passando por problemas financeiros atuais; $29,2 \%$ passaram por conflitos matrimoniais; $45 \%$ afirmaram já ter sofrido algum tipo de violência emocional ou física pelo parceiro ou alguém próximo;

Ao analisar 644

recém-nascidos de

mães adolescentes,

observou-se que

97,2\% dos partos

tiveram apresentação

cefálica, mas

cerca de $61 \%$ dos

casos analisados

necessitaram realizar

o parto cesáreo.

Além disso, a partir

do diagnóstico

pediátrico do

prontuário, cerca

de $10 \%$ dos recém-

nascidos eram

pequenos para idade

gestacional. e 7\% relataram já ter sofrido uma violência física durante a gravidez. ${ }^{16}$

Quanto às principais figuras de apoio da adolescente durante a gravidez, identifica-se que as mães das adolescentes são as que realizam o amparo físico e psicológico destas. Mesmo com a presença do companheiro, do pai da gestante e de outras figuras femininas, a mãe da adolescente se sobressai, ou seja, acaba sendo mais participativa na vida das filhas jovens do que as demais pessoas do convívio. ${ }^{17}$

Diante disso e considerando que a adolescente está em fase de crescimento e desenvolvimento biopsicossocial, torna-se necessário que esta receba informações dos profissionais de saúde que contribuam com seu bem-estar físico e emocional. A equipe de saúde deve orientar a adolescente sobre aspectos da gravidez, nutrição e cuidados com o recém-nascido. É necessário também testificar a importância do vínculo mãe-pai e bebê, a importância do aleitamento materno, vacinação da criança, prevenção de acidentes na infância, acompanhamento do crescimento e desenvolvimento e outros. Além disso, é imprescindível que haja uma orientação para adolescentes puérperas quanto ao planejamento reprodutivo e adesão de métodos contraceptivos para evitar uma nova gravidez a curto prazo. ${ }^{18-20}$

O pré-natal é de extrema importância para o cuidado com a gestante adolescente. Diante disso, adolescentes menores os 15 anos necessitam de uma atenção especial por apresentarem maiores riscos materno-fetal. ${ }^{18}$

Portanto, é necessária uma atenção especial durante a assistência ao pré-natal, uma vez que a ocorrência de uma assistência inadequada pode resultar em maior chance de nascimentos de crianças prematuras e de baixo peso, bem como de outras complicações maternas e fetais. ${ }^{18,21-22}$

\section{CONCLUSÃO}

Os achados da literatura apontam uma leve queda da gravidez na adolescência no Brasil, mas os números ainda são altos. Apesar do entendimento dos profissionais de saúde sobre a importância de programas de educação sexual para as famílias, falta ainda um acolhimento mais eficiente com 
o público jovem. O diálogo aberto precisa ser valorizado e estimulado.
Portanto, o acesso justo e de qualidade a educação pode ajudar os jovens a obter um olhar di- ferente do futuro e priorizar a busca por uma carreira profissional valorizando suas habilidades.

\section{REFERÊNCIAS}

1. Santos JLR, Aguiar RS. Cuidado de enfermagem aos adolescentes em cumprimento de medida socioeducativa. Rev Cereus [Internet]. 2020 [Acesso em 10 Nov 2020];12(1):184- 92. doi: 10.18605/2175-7275/cereus.v12n1p184-192

2. Alves LS, Aguiar RS. Saúde sexual e infecções sexualmente transmissíveis na adolescência: uma revisão integrativa. Nursing (São Paulo) [Internet]. 2020 [Acesso em 10 Nov 2020];23(263):368387. doi: 10.36489/nursing.2020v23i263p3683-3687

3. Bock AMB. A adolescência como construção social: estudo sobre livros destinados a pais e educadores. Psicol Esc Educ [Internet]. 2007 [Acesso em 10 Nov 2020];11(1):63-76. doi: 10.1590/ S1413-85572007000100007

4. Queiroz VR, Almeida JM. Sexualidade na adolescência: potencialidades e dificuldades dos professores de ensino médio de uma escola estadual de Sorocaba. Rev Fac Cienc Med Sorocaba [Internet]. 2017 [Acesso em 10 Nov 2020];19(4):209-14. Disponível em: http://ken.pucsp.br/RFCMS/article/view/31788/pdf

5. Fernandes DER, Medeiros M, Santos WS, Santos MG. Produção científica de enfermagem sobre a gravidez na adolescência: revisão integrativa. Aquichan [Internet]. 2020 [Acesso em 10 Nov 2020];20(2):e2025. doi: 10.5294/aqui.2020.20.2.5

6. Oliveira EFV, Gama SGN, Silva CMFP. Gravidez na adolescência e outros fatores de risco para mortalidade fetal e infantil no município do Rio de Janeiro, Brasil. Cad Saúde Pública [Internet]. 2010 [Acesso em 10 Nov 2020];26(3);567-78. doi: 10.1590/S0102$311 \times 2010000300014$

7. Fonseca MF, Núñez DF, Rodríguez MH, Reúna RR, Paneque TA. Intervenção educativa na gravidez na adolescência. CMF \# 37. Niquero. Multimed [Internet]. 2019 [Acesso em 17 Out 2020]; 23(4):731-43. Disponivel em: http://scielo.sld.cu/pdf/mmed/ v23n4/1028-4818-mmed-23-04-731.pdf

8. Rosaneli CF, Costa NB, Sutile VM. Proteção à vida e à saúde da gravidez na adolescência sob o olhar da bioética. Physis: Rev Saúde Coletiva [Internet]. 2020 [Acesso em 17 Out 2020];31(1):e300114. doi: 10.1590/S0103-73312020300114

9. Rother ET. Revisão sistemática $X$ revisão narrativa. Acta Paul Enferm [Internet]. 2007 [Acesso em 20 Abr 2021];20(2). Disponivel em: scielo.br/pdf/ape/v20n2/a01v20n2.pdf

10. Monteiro DLM, Martins JAFS, Rodrigues NCP, Miranda FRD, Lacerda IMS, Souza FM et al. Adolescent pregnancy trends in the last decade. Rev Assoc Med Bras [Internet]. 2019 [Acesso em 17 Out 2020];65(9):1209-15. doi: 10.1590/1806-9282.65.9.1209

11. Pinheiro YT, Pereira NH, Freitas GDM. Fatores associados à gravidez em adolescentes de um município do nordeste do Brasil. Cad Saúde Colet [Internet]. 2019 [Acesso em 17 Out 2020];27(4):363-67. doi: 10.1590/1414-462X201900040364

12. Santos GHN, Martins MG, Sousa MS, Batalha SJC. Impacto da idade materna sobre os resultados perinatais e via de parto. Rev Bras Ginecol Obstet [Internet]. 2009 [Acesso em 17 Out
2020];31(7):326-34. doi: 10.1590/S0100-72031009000700002

13. Bacelar EB, Costa COM, Gama SGN, Amaral MTR, Almeida AHV. Fatores associados à Síndrome Hipertensiva Específica da Gestação em puérperas adolescentes e adultas jovens da região Nordeste do Brasil: análise múltipla em modelos hierárquicos. Rev Bras Saude Mater Infant [Internet]. 2017 [Acesso em 17 Out 2020];17(4):683-91. doi: 10.1590/1806-93042017000400004

14. Silva MP, Santos ZMSA, Nascimento RO, Fonteles JL. Avaliação das condutas de prevenção da Síndrome Hipertensiva Específica da Gravidez entre adolescentes. Rev Rene [Internet]. 2010 [Acesso em 17 Out 2020];11(4):57-65. Disponível em: http://www.periodicos.ufc.br/rene/article/view/4595/3444

15. Parra EJL, Herrada CHL, Parra DML. El recién-nascido de madre adolescente. Rev Chil Obstet Ginecol [Internet]. 2018 [Acesso em 17 Out 2020];83(6):559-66. Disponivel em: https://scielo.conicyt.cl/scielo.php?script=sci_arttext\&pid=S0717-75262018000600559\&Ing=pt\&tlng=es

16. Pereira PK, Lovisi GM, Lima LA, Legay LF. Complicações obstétricas, eventos estressantes, violência e depressão durante a gravidez em adolescentes atendidas em unidade básica de saúde. Rev Psiquiatr Cli [Internet]. 2010 [Acesso em 17 Out 2020];37(5):216-22. Disponivel em: https://www.scielo.br/pdf/ rpc/v37n5/a06v37n5.pdf

17. Frizzo GB, Martins LWF, Silva EXL, Piccinini CA, Diehl AMP. Maternidade adolescente: a matriz de apoio e o contexto de depressão pós-parto. Psic Teor e Pesq [Internet]. 2019 [Acesso em 17 Out 2020];35:e3533. doi: 10.1590/0102.3772e3533

18. Almeida AHV, Costa COM, Gama SGN, Amaral MTR, Vieira GP. Baixo peso ao nascer em adolescentes e adultas jovens na região nordeste do Brasil. Rev Bras Saude Matern Infant [Internet]. 2014 [Acesso em 17 Out 2020];14(3):279-86. doi: 10.1590/S151938292014000300009

19. Aguiar RS, Araújo MAB, Costa MA, Aguiar N. Percepção de mulheres sobre o acolhimento oferecido pelo enfermeiro no pré-natal. Cogitare Enferm [Internet]. 2013 [Acesso em 17 Out 2020];18(3):527-31. doi: 10.5380/ce.v18i4.34933

20. Aguiar RS, Araújo MAB, Costa MA, Aguiar N. Orientações de enfermagem nas adaptações fisiológicas da gestação. Cogitare Enferm [Internet]. 2013 [Acesso em 17 Out 2020];18(4):756-60. doi: 10.5380/ce.v18i3.33567

21. Carniel EF, Zanolli ML, Almeida CAA, Morcilio AM. Características das mães adolescentes e de seus recém-nascidos e fatores de risco para a gravidez na adolescência em Campinas, SP, Brasil. Rev Bras Saude Matern Infant [Internet]. 2006 [Acesso em 17 Out 2020];6(4):419-26. doi: 10.1590/S1519-38292006000400009

22. Silva JLCP, Surita FGC. Idade materna: resultados perinatais e via de parto. Rev Bras Ginecol Obstet [Internet]. 2009 [Acesso em 17 Out 2020];31(7):321-25. Disponivel em: https://www.scielo. br/pdf/rbgo/v31n7/v31n7a01.pdf 